\title{
CIRROSIS HEPATICA EN LACTANTE: UNA FORMA DE PRESENTACION DE LA TIROSINEMIA
}

Drs. Rodolfo Mardones $\boldsymbol{R}^{(1)}$, Roberto Mondaca $A^{(1)}$, Ricardo Ávila $\boldsymbol{R}^{(2)}$.

1. Servicio de Radiología. Hospital Roberto del Río.

2. Servicio de Anatomía Patológica. Hospital Roberto del Río.

Abstract: We present two documented cases of patients with Tyrosinemia type I (Hepatorenal Tyrosinemia) in infants. The most constant imaging findings in target organs: Liver (Hepatic Cirrhosis), Kidneys (Nefromegaly) are described and compared with pathological findings in one case. In the presence of confusing clinical manifestations, radiological findings of hepatic cirrhosis in infants associated with renal involvement are almost diagnostic of this entity. Key words: Hepatic Cirrosis, Tyrosinemia type I, Hepatorenal Tyrosinemia.

Resumen: Se presentan 2 casos documentados de lactantes portadores de Tirosinemia tipo I (Hepatorenal). Se describen los hallazgos imagenológicos principales de ella en los órganos blanco: Hígado (Cirrosis Hepática) y riñones (Nefromegalia) y se confrontan con los de la anatomía patológica en un caso.

En un lactante, con un cuadro clínico poco claro, el hallazgo imagenológico de cirrosis hepática sumado a un compromiso renal, deben hacer plantear el diagnóstico de tirosinemia.

Palabras claves: Cirrosis hepática, Tirosinemia tipo I, Tirosinemia Hepatorenal.

\section{Introducción}

La tirosinemia es una grave enfermedad hereditaria de baja prevalencia que afecta el metabolismo de los aminoácidos. Una de sus formas de presentación compromete principalmente el hígado y los riñones. El conocimiento de los hallazgos radiológicos más constantes de esta entidad, permiten al radiólogo plantear la posibilidad diagnóstica.

Mardones $\boldsymbol{R}$, y cols. Cirrosis hepática en lactante: Una forma de presentación de la tirosinemia. Rev Chil Radiol 2002; 8: 164-167.

Correspondencia: Dr. Rodolfo Mardones R. Hospital Roberto del Río. Profesor Zañartu 1085. Independencia. Santiago-Chile.E-mail: romardones@yahoo.com
Se presentan dos casos documentados, de pacientes portadores de tirosinemia tipo I (hepatorrenal), en quienes los hallazgos imagenológicos orientaron el diagnóstico.

\section{Casos Clínicos}

Caso 1: Lactante de 9 meses de edad, del sexo femenino, con cuadro de 3 meses de evolución, caracterizado por: desnutrición calórico-proteica severa, raquitismo refractario a vitamina $D$, acidosis metabólica y hepatoesplenomegalia.

Se efectuó estudio ultrasonográfico abdominal en el que destacó la presencia de hepatomegalia, con parénquima de estructura heterogénea y múltiples nódulos hiperecogénicos de hasta $1.8 \mathrm{~cm}$. Estos nódulos presentaban un fino halo hipoecogénico y no se detectó vascularización al ser evaluados con modo Doppler (Figuras 1a,1b). Los riñones se apreciaban aumentados de volumen y con aumento de ecogenicidad a nivel cortical (Figura 1c).

Se complementó el estudio con tomografía axial computarizada de abdomen, que demostró hepatomegalia con nódulos hipodensos de diferentes tamaños, algunos bien definidos, cuya densidad no aumentó después de la inyección de medio de contraste endovenoso (Figuras 2a, 2b). Los riñones se visualizaron aumentados de volumen y con buena captación del contraste endovenoso, aunque con retardo en su eliminación (Figura 2b).

El paciente falleció y en el examen anátomo-patológico se comprobó a la macroscopía: hígado aumentado de tamaño, de coloración amarillenta con múltiples nódulos de regeneración menores de $1 \mathrm{~cm}$ de diámetro (Figura 3).

Caso 2: Paciente de sexo masculino de un año de edad con cuadro clínico de desnutrición severa progresiva, raquitismo y hepatoesplenomegalia.

El examen ultrasonográfico demostró hepatomegalia difusa de aspecto multinodular (Figura 4a), los riñones eran grandes e hiperecogénicos, sugerentes de nefropatía médica (Figuras $4 b$ ). En este 

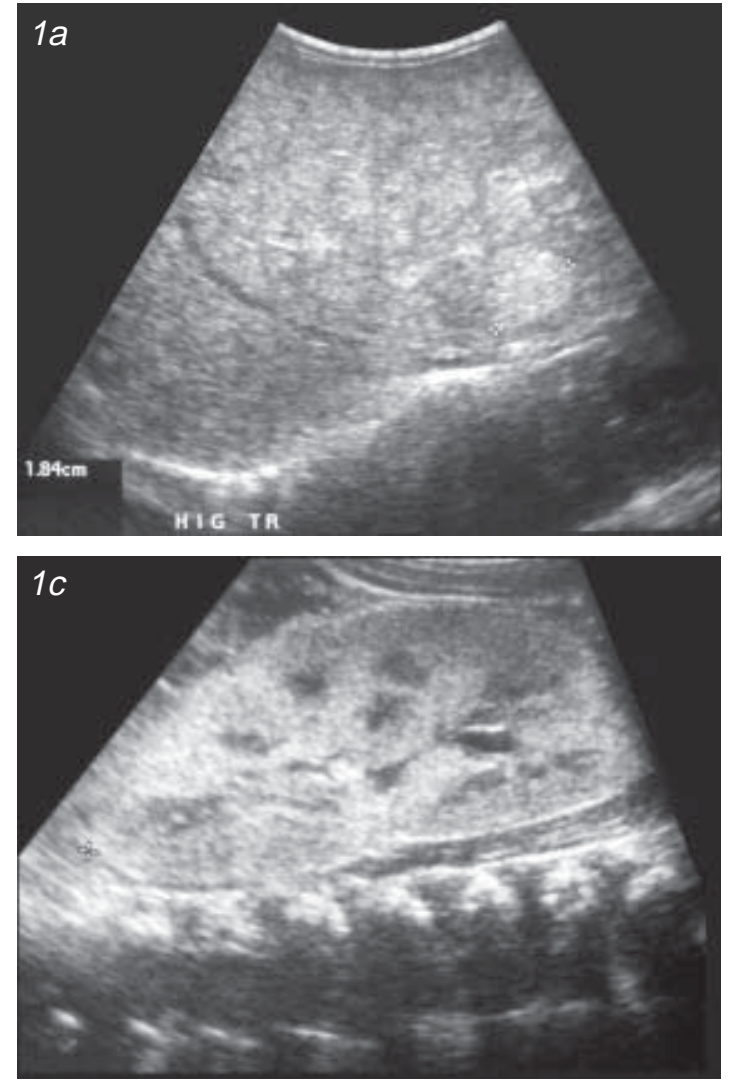

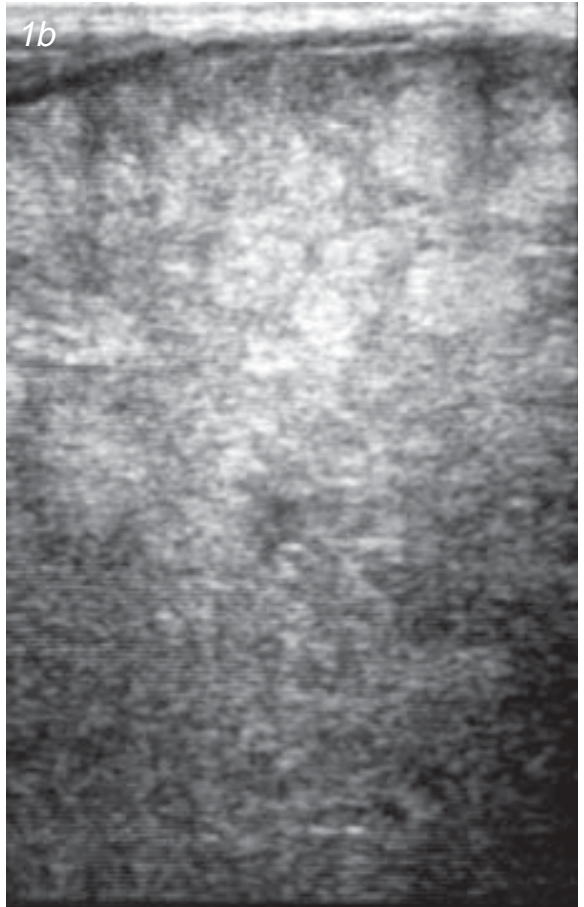

Figura 1 a, b, c. Caso 1. Ultrasonografía abdominal. a:. Hígado. Múltiples nódulos hepáticos hiperecogenicos. Uno de ellos de $1.84 \mathrm{~cm}$. entre signos (+). b: Detalle de nódulos con transductor de alta resolución. c: Riñón demostrando aumento de volumen y ecogenicidad.
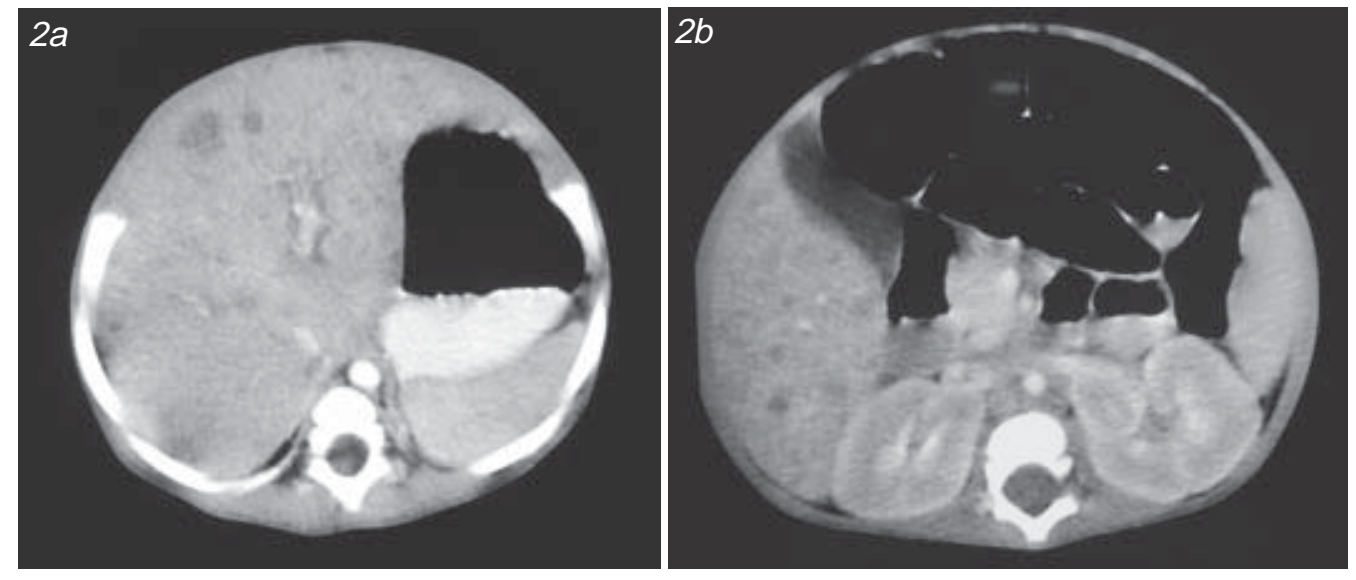

Figura 2 a,b. Caso 1. TAC de abdomen. a: Hígado con nódulos hipodensos. b: Riñones aumentado de volumen con impregnación cortical persistente $y$ retardo en la eliminación del contraste.
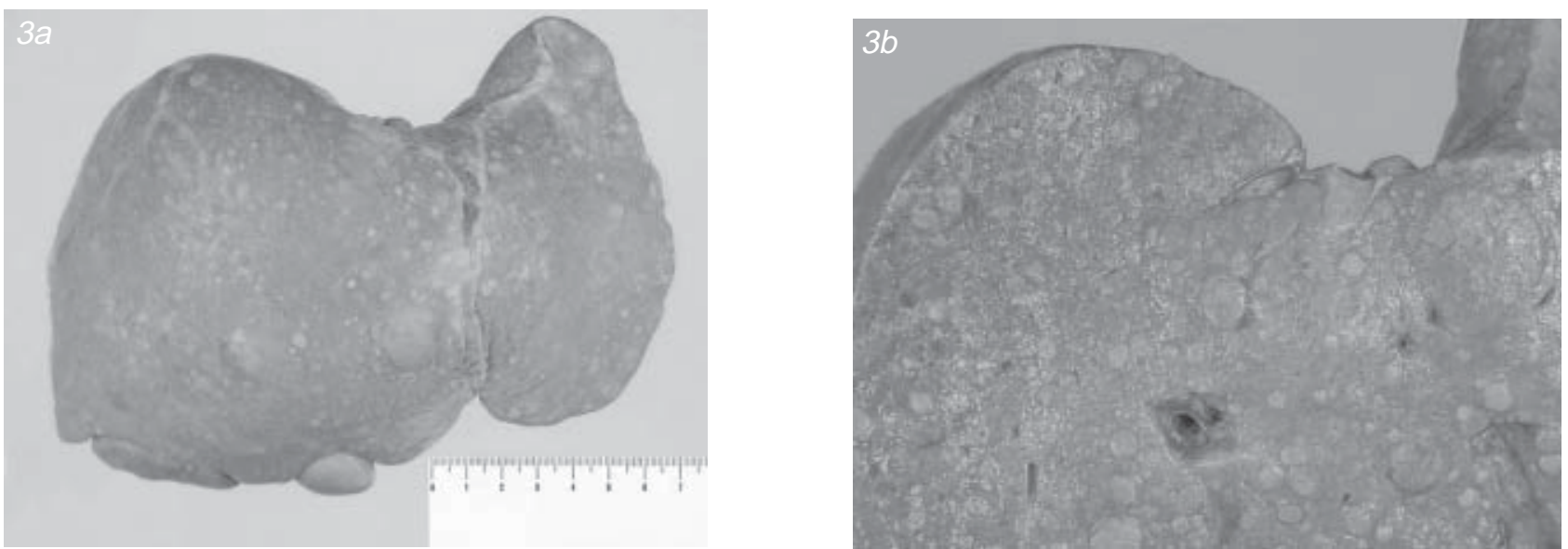

Figura 3 a,b. Caso 1. Pieza anatómica. a: Hígado visión macroscópica (superficie externa). b: Superficie interna (corte anatómico). Destacan múltiples nódulos amarillentos consistentes con Cirrosis macro y micronodular. 
paciente además se efectuó estudio radiográfico de antebrazo izquierdo (Figura 5), que demostró signos de raquitismo, propio de esta entidad clínica.

Este paciente también falleció efectuándose sólo biopsia hepática.
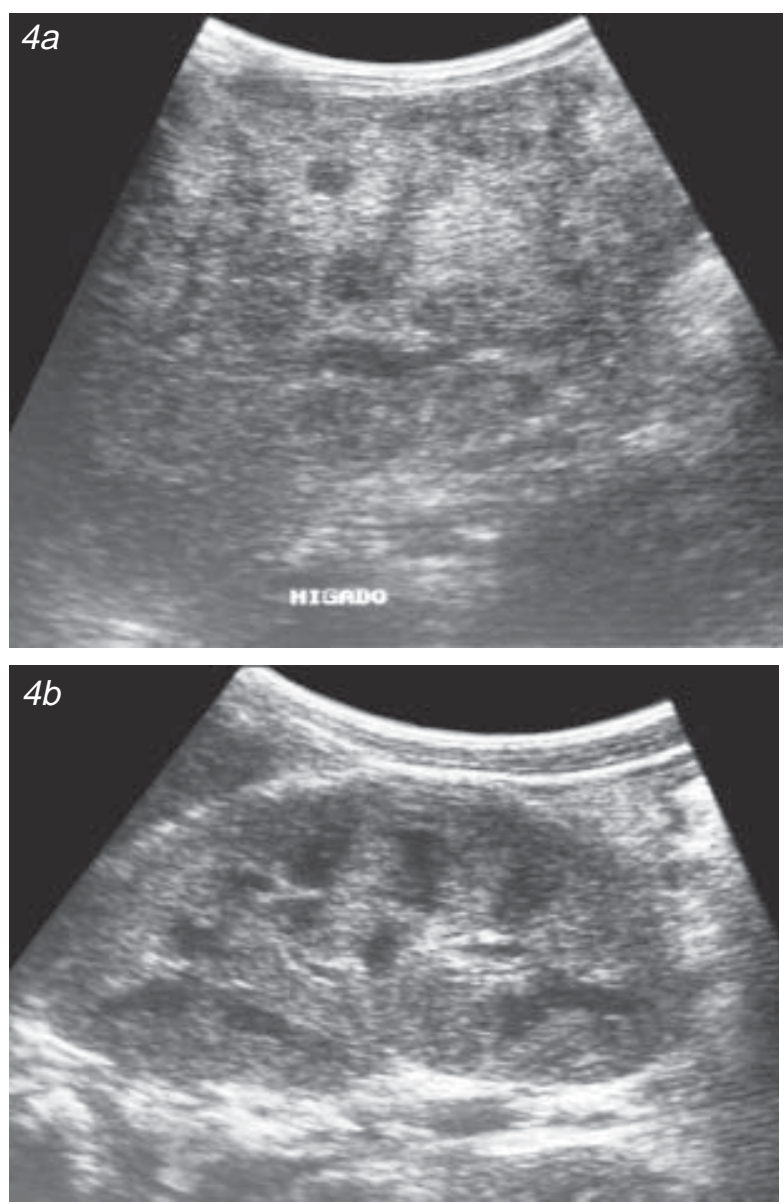

Figura 4 a,b. Caso 2. Ultrasonografía de abdomen. a: Hígado destacando la presencia de nódulos hiperecogénicos. b: Riñón izquierdo aumentado de volumen y con cortical hiperecogenica. Su tamaño es de $9.4 \mathrm{~cm}$.

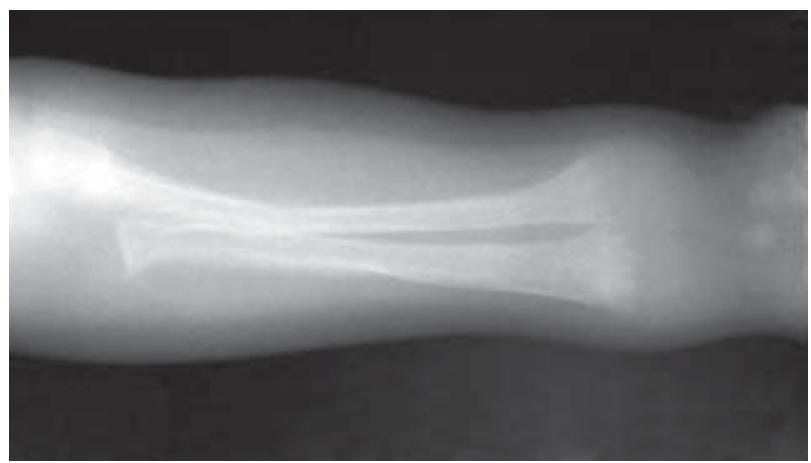

Figura 5. Caso 2. Radiografía de antebrazo izquierdo, demuestra a nivel de metáfisis distal de cubito y radio ensanchamiento y deshilachamiento, signos sugerentes de raquitismo.
En ambos casos el diagnóstico se confirmó mediante la medición de actividad enzimática (fumarilacetoacetatohidrolasa) en fibroblastos.

\section{Discusión}

La tirosinemia es una enfermedad hereditaria poco frecuente con una prevalencia mundial 1/ 100.000 a 1/120.000, que pertenece al grupo de las enfermedades hereditarias del catabolismo de los aminoácidos $^{(1)}$.

Se describen 3 subtipos que varían según la alteración enzimática que presentan: a) Tipo I determinada por el déficit de la fumarilacetoacetato hidrolasa $(\mathrm{FAH})$ que es la última enzima en la degradación de la tirosina, b) Tipo II que esta determinada por el déficit de tirosina aminotransferasa, fracción citosólica, c) Tipo III que ocurre por el déficit de la 4hidroxifenilpiruvatodioxigenasa. Todos ellos presentan una herencia autosómica recesiva ${ }^{(1)}$.

La tirosinemia tipo I es el defecto genético más común y el más grave, dentro del espectro de anomalías en la degradación de la tirosina(2).

Está descrito en la literatura, una alta incidencia de tirosinemia tipo I en niños de la región de Saguenay-Lac St. Jean en la provincia de Québec, Canadá con una incidencia de 1 de cada 1846 recién nacidos ${ }^{(1)}$.

El déficit de $\mathrm{FAH}$, se acompaña de una moderada elevación de la tirosina sérica, lo que se asocia a severo compromiso de hígado, riñones y sistema nervioso central. Esta afectación sería provocada por la acumulación de los metabolitos intermediarios de la tirosina, especialmente de succinilacetona, en los tejidos $^{(1)}$.

La historia natural de la enfermedad, muestra desarrollo precoz de cirrosis hepática (macromicronodular) con un alta tasa de progresión a hepatocarcinoma, lo que se piensa estaría determinado por el efecto mutagénico e influencias en el ciclo celular causadas por la acumulación de metabolitos en el parénquima hepático ${ }^{(3,4)}$.

El daño a nivel renal, evidenciado por las imágenes, no estaría provocado por depósito de metabolitos o edema. La única anomalía estructural que podría explicarlo es la dilatación de los túbulos proximales demostrada en el estudio histológico ${ }^{(5)}$. Ello puede también explicar el aumento de ecogenicidad evidenciado en ambos casos.

Desde el punto de vista clínico, los pacientes pueden permanecer asintomáticos durante el primer año de vida o presentar cuadros de desnutrición severa, hepatoesplenomegalia, fiebre, irritabilidad, vómitos, etc. A menor edad de aparición de los síntomas, mayor será la gravedad del cuadro clínico ${ }^{(1)}$.

Aun cuando, el diagnóstico de esta entidad se basa fundamentalmente en los hallazgos clínicos y de laboratorio fundamentalmente por la determina- 
ción de la actividad de FAH en biopsia hepática ${ }^{(6)}$, en el caso de la tirosinemia tipo I (tirosinemia hepatorrenal), las manifestaciones imagenológicas en los órganos blanco: Hígado (Cirrosis Hepática) y riñones (nefromegalia), son constantemente, descritas en la literatura ${ }^{(5,7)}$, y pueden dar elementos claves para el diagnóstico oportuno de esta enfermedad.

\section{Conclusión}

La tirosinemia es una entidad clínica poco frecuente y cuyo diagnóstico se basa fundamentalmente en los parámetros clínicos y de laboratorio. Sin embargo, el hallazgo imagenológico de cirrosis hepática, sumado al compromiso renal descrito, en un lactante con un cuadro clínico poco claro, debe hacer pensar en el diagnóstico de tirosinemia hepatorenal.

\section{Bibliografía}

1. Behrman, Kliegman, Jenson. Nelson Textbook of Pediatrics 16th Edition, 2000 W.B:Saunders Company.

2. Grompe M. The pathophysiology and treatment of hereditary tyrosinemia type1. Semin Liver Dis. 2001; 21:563-71.

3. Russo P, O'Regan S. Visceral pathology of hereditary tyrosinemia type I. Am J Hum Genet 1990 ; 47:317-24.

4. Russo PA, Mitchel GA, Tanguay RM. Tyrosinemia a review. Pediatr Dev Pathol. 2001; 4:212-21.

5. Forget $\mathrm{S}$, Patriquin $\mathrm{H}$, Dubois J. et al. The kidney in children with tyrosinemia: sonographic, CT and biochemical findings. Pediatr Radiol 1999; 29:104-108.

6. Burton B. Inborn Errors of Metabolism in Infancy: A guide to diagnosis. Pediatrics 1998; 102 (6).

7. Pediatric case of the day. Hepatorrenal Tyrosinemia (Tyrosinemia Type I). Radiographics 1996; 16 :12211224. 